\title{
Efficacy of Platelet Gel in Children with Stomatitis during Chemotherapy
}

Katarzyna Albrecht ${ }^{1}$, Paweł Łaguna ${ }^{1}$, Elżbieta Lachert² ${ }^{2}$ Karolina Gawle-Krawczyk ${ }^{1}$

1 Department of Pediatrics, Hematology, and Oncology, The Medical University of Warsaw, Warsaw, Poland

2 Department of Transfusion Medicine, Institute of Hematology and Transfusion Medicine, Warsaw, Poland

\begin{abstract}
Introduction. Stomatitis (oral mucositis) is a very serious adverse effect of anticancer therapy, and it may exacerbate treatment outcomes as well as prognoses. Stomatitis is associated with pain during chewing and swallowing, causing discomfort and interfering with eating. It can also lead to weight loss and a delay in child growth and development. None of the numerous therapies used for stomatitis management can be considered universal or sufficiently effective. Allogeneic platelet gel, a formulation that adheres closely to mucosal lesions and is rich in growth factors, may be an effective treatment for accelerating the erosion-healing process.

Methods. A prospective, open-label study on the efficacy of platelet gel used for the treatment of stomatitis in children during chemotherapy. Platelet gel was applied to oral mucosal lesions four times a day.

Results. 28 patients with Grade II and Grade III stomatitis were enrolled in the study. The first day after applying platelet gel, $93 \%$ of patients reported relief from pain. Within 4 to 5 days, one degree of mucosal lesion regression was reported in $89 \%$ of patients, indicating a 3 to 5 day reduction in therapy compared to our previous experience. In only two patients did we observe mild adverse events in the form of a burning sensation.

Conclusion. Platelet gel contributed to both pain reduction and acceleration of the oral mucosa healing process. It is a Keywords safe and effective therapy for children with stomatitis induced by chemotherapy.
\end{abstract}

leukemia $\bullet$ pediatrics $\bullet$ periodontal disease $\bullet$ platelet-rich plasma

Received: 18.10.2020, Accepted: 15.05.2021

\section{Introduction}

Stomatitis (oral mucositis) is one of the most serious adverse effects of anticancer therapy, occurring in 40-80\% of patients undergoing cancer therapy. It is an inflammatory process affecting the oral mucosa and lips, and manifests as redness and soreness either with or without oral ulceration [1]. The severity and extent of stomatitis (Table 1) can vary according to treatment type and the condition of the patient. Oral inflammation may be affected by the type of therapy (i.e., chemotherapy or radiotherapy), medication, dosage, administration route, and number of chemotherapy cycles, or the total dose and extent of the area subjected to radiation. The incidence of stomatitis in patients is approximately $40 \%$ for chemotherapy, $80 \%$ for radiotherapy, and over $75 \%$ after bone-marrow transplants [3, 4]. A higher risk for the condition is associated with an increased number of chemotherapy cycles, and administration of methotrexate, doxorubicin, bleomycin, cisplatin, carboplatin, busulfan, 5-fluorouracil, mTOR inhibitor, and tyrosine kinase inhibitors. Patient-related risk factors include cancer type, age, oral hygiene, body-mass index, gender, coexisting salivary-gland dysfunction, comorbidities (e.g., diabetes, Addison's disease, and psoriasis), and genetic factors such as gene polymorphisms that affect drug metabolism $[3,5,6]$.

The main problem for patients with stomatitis is the pain that accompanies chewing and swallowing. This significantly impairs their quality of life and comfort, interferes with food intake, and can lead to weight loss, cachexia, and the retardation of growth and development in children. Due to the condition, patients often require opioid analgesia and parenteral nutrition, with additional serious side effects. For immunosuppressed and myelosuppressed patients, mouth ulcers are gateways for lifethreatening infections and sepsis. Moreover, stomatitis prolongs the interval between consecutive stages of chemotherapy during cancer treatment, worsening prognoses and reducing survival rates [4]. 
Table 1. The WHO scale of oral inflammation severity [2]

\begin{tabular}{cc}
\hline Grade & Criteria \\
\hline I & No changes \\
II & Pain, redness \\
III & Ulcers, solid meals possible \\
IV & Ulcers, liquid diet only \\
\hline
\end{tabular}

In clinical practice, in addition to proper oral hygiene and a balanced diet, many measures are used to prevent inflammation and reduce pain and discomfort [2, 7]. Firstline therapies for stomatitis are topical agents that protect the surface of the damaged mucosa and support its regeneration, topically applied analgesics, antifungals, anti-inflammatories, and antiseptic drugs and topical corticosteroids. In some cases, the use of systemic steroids, antibiotics, antifungal and antiviral drugs, as well as vitamin and trace-element supplements have proved helpful. According to a Cochrane Library review, other beneficial treatments include aloe vera, amifostine, cryotherapy, granulocyte-colony stimulating factor, intravenous glutamine, honey, keratinocyte growth factor, laser therapy, polymyxin and sucralfate, but none of these are considered universal or sufficiently effective [8].

Implementation of a completely effective therapy for stomatitis is a constant challenge due to its complexity, poorly understood pathogenesis, and unsatisfactory outcomes of commonly available methods for treatment and prevention. The introduction of a new allogeneic blood product, platelet gel (PG), appears to be a promising solution. PG is obtained from platelet-rich plasma and consists of a platelet concentrate (PC) embedded within a semi-solid network of polymerized fibrin [9]. Platelet activation results in the release of therapeutically active growth factors from their alpha granules and dense granules. Moreover, the concentrated fibrinogen increases tissue adhesion, protecting tissue from mechanical and chemical factors, and strengthens the local/ topical effect of the growth factors. To date, the effectiveness of $P G$ in stomatitis cases has been reported in children during anticancer therapy, and in adults with radiation-induced stomatitis $[1,10,11]$. The aim of the present study was to evaluate the efficacy of $P G$ in children with stomatitis during chemotherapy.

\section{Materials and Methods}

\section{Study Design}

A prospective, open-label study of the efficacy of platelet gel $(P G)$ in children with stomatitis during chemotherapy.

\section{Participants}

The study included children (0-18 years old) who developed stomatitis during cancer therapy in the Department of Pediatrics, Hematology, and Oncology at the Medical University of Warsaw from July 2018 to March 2019. Diagnosis of stomatitis was based on clinical examination. The degree of inflammation was assessed according to a World Health Organization (WHO) scale (Table 1). Inclusion criteria were stomatitis Grades II and III. Patients with Grade IV stomatitis did not qualify due to severe pain and an inability to open the mouth sufficiently, and those with Grade I did not qualify because the pathology was mild, with no indication for a more powerful intervention.

\section{Platelet Gel}

Allogeneic PG was prepared at the Institute of Hematology and Transfusion Medicine from PCs collected at the Regional Blood Transfusion Center in Warsaw. PCs were obtained using a cell separator (AMICUS ${ }^{\circledR}$, Fresenius Kabi AG, Bad Homburg vor der Höhe, Germany) according to Polish regulations, and were subjected to a pathogen-inactivation procedure (Mirasol ${ }^{\otimes}$ PRT, Terumo BCT, Japan; and the Intercept Blood System $^{\circledR}$, Cerus Corporation, Concord, USA). All procedures were performed in a closed system using a sterile connection device (TSCD ${ }^{\circledR}$, Terumo BCT, Japan). PG was immediately frozen for on-site storage, or transported frozen $\left(-18^{\circ} \mathrm{C}\right)$. PG was prepared for patient application immediately before use. Freezing and thawing activated platelet cells and released growth factors. Simple preparation of the platelet gel was carried out in a closed system, which additionally increased the microbiological safety of PG.

\section{Intervention}

In the study group, $A B O$ group-compatible PG was applied in $2 \mathrm{ml}$ doses (with cotton swabs) to mucosal lesions four times per day. All patients also received topical agents such as Mix No. 1 (3\% hydrogen peroxide, $0.1 \%$ lidocaine, nystatin, and dolomite), nystatin, miconazole, and an herbal mouthwash containing panthenol and allantoin. Analgesics (e.g., acetaminophen, tramadol, and morphine) were administered to patients who developed pain, dysphagia, and severe stomatitis. Additionally, most patients received intravenous antifungal agents (e.g., fluconazole, micafungin, 
or amphotericin B) due to agranulocytosis and the risk of developing a fungal infection.

\section{Outcomes}

The primary endpoint of the study was stomatitis regression by at least one degree on the WHO classification scale. Regression time was assessed with an accuracy of one day. Secondary endpoints included relief from pain, degree of pain reduction, and PG safety. Assessment of pain intensity was dependent on patient age and developmental level using analog or numeric scales, or subjective opinions of caregivers for infants and younger children. Safety was assessed by the incidence of adverse reactions reported by patients and/or caregivers.

\section{Results}

The study enrolled 28 patients ( 20 males and 8 females) aged 8 months to 17 years (median 10.4); 19 of them were treated for acute lymphoblastic leukemia, one for acute myeloid leukemia, one for myelodysplastic syndrome, and seven for Burkitt's lymphoma. In all patients, stomatitis developed during the period of pancytopenia after a course of chemotherapy (average 3-4 days after the end of intensive chemotherapy). In these patients, the number of leukocytes was below $0.5 \times$ $10 \%$ and was accompanied by agranulocytosis. In the study group, 26 patients were diagnosed with Grade III stomatitis, and two with Grade II.

In $93 \%$ of patients ( $n=26$ ), the application of PG brought relief, reduced pain, and reduced any burning sensation after the first day of application. In $89 \%$ of patients ( $n=25$ ), there was a significant improvement in the appearance of mucous membranes and inflammatory lesion regressions within 4-5 days: regressions from Grade III to Grade II in 23 cases, and from II to I in two cases. One patient, with WHO Grade III stomatitis, showed regression after 8 days. In two patients (7\%), the application of PG caused a worsening of symptoms with increased pain and a sensation of burning. In these patients (4 and 9 years old), after three attempts the use of $P G$ was discontinued. No progression to the higher grade of stomatitis was observed in any patient.

\section{Discussion}

\section{Stomatitis Healing}

Chemotherapeutics trigger an inflammatory cascade, have direct and destructive effects on the epithelium and its DNA, and may also cause the formation of free radicals. This inflammation process not only applies to the epithelium, but also to the submucosal layer and the intercellular matrix [4, 12]. Moreover, mucosal lesions are constantly exposed to irritation associated with food intake, which explains why the mucosa healing process in stomatitis is prolonged. Based on the literature, regression of stomatitis takes approximately two weeks [4, 12, 13]. We conducted a retrospective analysis of stomatitis cases in pediatric patients treated with chemotherapy (similar protocols to those used in our study group) at our center during the two years preceding this study (data not published). The average duration of Grade II and III stomatitis before regression by one grade was 7-10 days. Progression to a higher grade of stomatitis was also observed in many of those patients. In the present study, no cases of progression were seen, and $89 \%$ of patients experienced a reduction in stomatitis severity within 4-5 days. Therefore, the use of PG shortened the time of mucosal-lesion treatment by 3-5 days and may have prevented stomatitis progression. These results are consistent with previously published data $[1,10,11]$.

\section{Pain Reduction}

Previous studies have shown an improvement in the quality of life after $P G$ treatment in adult patients with stomatitis during anticancer therapy [10]. Here, PG was effective in reducing pain in the vast majority $(93 \%)$ of patients after the first day of use. Only in two patients did the therapy not bring pain relief, and these individuals complained of additional discomfort which resulted in termination of the therapy after three attempts. During the assessment of pain, by definition a subjective feeling, the possibility of a placebo effect cannot be excluded. In addition, the intensity of pain in the youngest patients was assessed by caregivers based only on observations, and their knowledge of the patients' reactions. These assessments may not have fully reflected actual feelings of discomfort. However, such a high percentage of pain relief is a cause for optimism, even taking into account a possible placebo effect. The specific use of placebo controls should be adopted in future studies.

\section{Safety}

The use of PG appears to be very safe. Only two patients reported repeated adverse reactions in the form of a burning sensation, which resulted in the termination of $P G$ treatment for them. No serious adverse events were reported, and PG was assessed as easy to use, even in very young children.

\section{Advantages and Limitations}

This was the first study to evaluate the efficacy of $P G$ in pediatric patients with stomatitis during chemotherapy. Its main disadvantages were the lack of any real-time controls, and the lack of a comparison to a placebo control, both of which can be incorporated into future research on PG usability. 
Conclusions

$P G$ reduced pain and accelerated the healing process of the oral mucosa. It was safe and should be considered an effective therapeutic method for pediatric patients with stomatitis during chemotherapy. These positive PG effects require confirmation in future randomized, placebo-controlled studies.

\section{Acknowledgments}

We are very grateful to Dr. Łukasz Dembiński for the valuable advice regarding manuscript preparation.

\section{Authors' Contributions}

K.A.: supervising the project, writing the manuscript, acquisition of data, literature review, analysis and interpretation of data, designing the figures; P.t.: research concept and design, supervising, writing the manuscript, drafting the article or revising it critically for important intellectual content; E.L.: research concept and design, writing the manuscript, final proofreading and approval of the version for publication; k.g.-k.: carrying out the experiments, acquisition of data.

\section{Conflict of Interest}

The authors have no potential conflicts of interest to declare.

\section{Statement of Ethics}

The study was approved by the Clinical Research Ethics Committee of the Institute of Hematology and Transfusion Medicine, Warsaw, Poland (number IHiT/25/2019). All parents, and patients over the age of 16 years, signed an informed-consent form.

\section{References}

[1] Aielli F., Giusti R., Rughetti A., dell'Orso L., Ficorella C., Porzio G.: Rapid resolution of refractory chemotherapy-induced oral mucositis with platelet gel-released supernatant in a pediatric cancer patient: A case report. J. Pain Symptom Manage, 2014; 48: e2-e4

[2] Bensinger W., Schubert M., Ang K.K., Brizel D., Brown E., Eilers J.G., Elting L., Mittal B.B., Schattner M.A., Spielberger R., et al.: NCCN Task Force Report. Prevention and management of mucositis in cancer care. J. Natl. Compr. Canc. Netw., 2008; 6, Suppl 1: S1-S21

[3] Epstein J.B., Thariat J., Bensadoun R.J., Baqrasch A., Murphy B.A., Kolnick L., Popplewell L., Maghami E.: Oral complications of cancer and cancer therapy: From cancer treatment to survivorship. CA Cancer J. Clin., 2012; 62: 400-422

[4] Parkhill A.L.: Oral mucositis and stomatitis associated with conventional and targeted anticancer therapy. J. Pharmacovigilance, 2013; 1: 112

[5] Pico J.L., Avila-Garavito A., Naccache P.: Mucositis: Its occurrence, consequences, and treatment in the oncology setting. Oncologist, 1998; 3: 446-451

[6] Raber-Durlacher J.E., Elad S., Barasch A.: Oral mucositis. Oral Oncol., 2010; 46: 452-456

[7] Chaveli-López B., Bagán-Sebastián J.V.: Treatment of oral mucositis due to chemotherapy. J. Clin. Exp. Dent., 2016; 8: e201-e209

[8] Worthington H.V., Clarkson J.E., Bryan G., Furness S., Glenny A.M., Littlewood A., McCabe M.G., Meyer S., Khalid T.: Interventions for preventing oral mucositis for patients with cancer receiving treatment. Cochrane Database Syst. Rev., 2011; 2011: CD000978

[9] Mazzucco L., Balbo V., Cattana E., Borzini P.: Platelet-rich plasma and platelet gel preparation using Plateltex. Vox Sang, 2008; 94: 202-208

[10] Bonfili P., Gravina G.L., Marampon F., Rughetti A., Di Staso M., Dell'Orso L., Vittorini F., Moro R., La Verghetta M.E., Parente S., et al.: Oral platelet gel supernatant plus supportive medical treatment versus supportive medical treatment in the management of radiation-induced oral mucositis: A matched explorative active control trial by propensity analysis. Am. J. Clin. Oncol., 2017; 40: 336-341

[11] Di Staso M., Rughetti A., Dell'Orso L., Marampon F., La Verghetta M.E., Parente S., Gravina G.L., Aielli F., Dolo V., Ruggieri V., et al.: Topical application of platelet supernatant gel in the management of radiotherapy-induced mucositis: A case report. Blood Transfus., 2014; 12: 107-110

[12] Squier C.A.: Oral complications of cancer therapies. Mucosal alterations. NCI Monogr., 1990: 169-172

[13] Sonis S.T.: Mucositis: The impact, biology and therapeutic opportunities of oral mucositis. Oral Oncol., 2009; 45: 1015-1020 